\title{
Antihypertensive Therapie - was darf's kosten?
}

\section{Die Hypertoniebehandlung ist kosteneffizient}

Die arterielle Hypertonie ist ein Risikofaktor für kardiovaskuläre Erkrankungen wie Myokardinfarkt, Herzinsuffizienz oder cerebrovaskulären Insult (CVI). Viele Studien haben den Nutzen einer antihypertensiven Behandlung bezüglich Verhinderung solcher Erkrankungen gezeigt. Dies trifft insbesondere für ACE-Hemmer, AngiotensinRezeptor Blocker (ARB, Sartane), Betablocker, Kalzium-Antagonisten und Thiazid-Diuretika zu (zusammengefasst in http://www.swisshypertension.ch/docs/guidelines_2009_d_leaflet.pdf). Sie werden zur Hypertoniebehandlung von der Schweizerischen Hypertoniegesellschaft als Mono- oder Kombinationstherapien empfohlen, wobei die Betablocker eher in den Hintergrund gerückt sind [1].

Auch bezüglich Kosteneffizienz lohnt sich eine Blutdruckbehandlung, wie in einer norwegischen Studie gezeigt werden konnte [2]; die Number Needed to Treat (NNT) bei Patienten mit mittelschwerer arterieller Hypertonie zur Verhinderung eines CVI beträgt 120 Patienten; die Behandlung eines CVI kostet in Norwegen 24000 Euro, falls schwere neurologische Folgeschäden auftreten, fallen zusätzlich bis 100000 Euro Pflegekosten pro Jahr an. Die Behandlung eines Myokardinfarkts kostet 20000 Euro, die Behandlung von Patienten mit Herzinsuffizienz kostet 3600 Euro jährlich. Demgegenüber kostet die Blutdruckbehandlung in Norwegen (Arztkosten und Medikamentenkosten) ca. 200 Euro jährlich. Bezüglich Kosteneffizienz der verschiedenen Stoffklassen gab es in dieser Studie keinen klaren Sieger.

\section{Auswahl von Antihypertensiva bezüglich Nebenwirkungsprofil und Co-Morbiditäten}

Nebenwirkungen in der medikamentösen Behandlung von Patienten mit arterieller Hypertonie sind uns Grundversorgern geläufig: Vor allem Reizhusten bei der Behandlung mit ACE-Hemmern (beobachtet häufiger als die publizierten 15-30\%), Müdigkeit bei Beta- blockern, Beinödeme bei Kalziumantagonisten und Dyselektrolytämien bei Thiaziden sind sehr häufig. Oft muss mehrmals gewechselt werden, bis eine gut wirksame und gut verträgliche Therapie gefunden wird. Niedrigdosierte Kombinationen sind additiv in der Wirkung aber nicht in den Nebenwirkungen und werden deshalb möglicherweise besser Vertragen als hochdosierte Einzelsubstanzen [3]. Wie oben erwähnt spielt die Wirkstoffklasse oder die Kombination bei der Primärprävention eine untergeordnete Rolle. Anders sieht es aus wenn Co-Morbiditäten oder eine Schwangerschaft vorliegen. Auch hier liegen Empfehlungen der Schweizerischen Hypertoniegesellschaft [1] vor:

- bei Koronarer Herzkrankheit: Betablocker, ACE-Hemmer, ARB und Kalziumantagonist

- bei Herzinsuffizienz: ACE-Hemmer, Diuretika, Betablocker, ARB, Aldosteron-Antagonist

- bei diabetischer Nephropathie: ACE-Hemmer, ARB, Reninhemmer - bei Asthma und COPD: keine Betablocker

- bei Diabetes: ACE-Hemmer, ARB, Kalziumantagonist

- bei Schwangerschaft: Alpha-Methyldopa, Labetalol, Dihydropyridin

\section{Vergleich bezüglich Kosten einer Blutdrucktherapie}

Noch vor wenigen Jahren wurde den Assistenzärztinnen und Assistenzärzten im Spital gelehrt, aus kostengründen ACE-Hemmer den teureren ARB vorzuziehen, und nur im Falle von Nebenwirkungen zu wechseln. Auch das Netzwerk Medix Schweiz hat diese Praxis in ihre Guidelines aufgenommen. Nun haben innert kurzer Zeit Losartan, Valsartan, Candesartan und Irbesartan den Patentschutz verloren, was zu massiven Preisreduktionen der Sartane geführt hat. In Tabelle 1 sind die Jahrestherapiekosten und die Effektivtät in der Blutdrucksenkung einiger Antihypertensiva zusammengestellt. Die Tabelle zeigt, dass sich das «Preis-Leistungsverhältnis» zugunsten

Tabelle 1

Auswahl verschiederer Antihypertensiva und Therapiekosten pro Jahr.

\begin{tabular}{|c|c|c|c|c|c|}
\hline $\begin{array}{l}\text { Wirkstoff } \\
\text { (Klasse) }\end{array}$ & Präparat & Mögliche Tagesdosis & $\begin{array}{l}\text { Preis/Jahr }{ }^{1} \\
\text { in CHF }\end{array}$ & $\begin{array}{l}\text { Mögliche Syst. } \\
\text { BD-Senkung }\end{array}$ & $\begin{array}{l}\text { Preis pro } 10 \mathrm{~mm} \mathrm{Hg} / \\
\text { Jahr in CHF }\end{array}$ \\
\hline $\begin{array}{l}\text { Lisinopril } \\
\text { (ACE-Hemmer) }\end{array}$ & $\begin{array}{l}\text { z.B. Lisinopril Actavis }{ }^{\circledR} \\
\text { Tbl. à } 20 \text { mg }\end{array}$ & $10 \mathrm{mg}$ & 103 & $9 \mathrm{~mm} \mathrm{Hg}^{2}$ & 114 \\
\hline $\begin{array}{l}\text { Valsartan } \\
\text { (ARB) }\end{array}$ & $\begin{array}{l}\text { z.B. Valsartan Spirig } \\
\text { Tbl. à } 160 \mathrm{mg}\end{array}$ & $80 \mathrm{mg}$ & 104 & $9 \mathrm{~mm} \mathrm{Hg}^{2}$ & 115 \\
\hline Aliskiren (DRI) & Rasilez $^{\circledR}$ & $300 \mathrm{mg}$ & 538 & $20 \mathrm{~mm} \mathrm{Hg}^{3}$ & 269 \\
\hline $\begin{array}{l}\text { Amldoipin } \\
\text { (Ca-Antagonist) }\end{array}$ & $\begin{array}{l}\text { z.B. Amlodipin Axapharm }{ }^{\circledR} \\
\text { Tbl. à } 10 \text { mg }\end{array}$ & $5 \mathrm{mg}$ & 121 & $9 \mathrm{~mm} \mathrm{Hg}^{2}$ & 134 \\
\hline $\begin{array}{l}\mathrm{HCT} \\
\text { (Thiazid-Diuretikum) }\end{array}$ & Esidrex $^{\circledR}$ Tbl. à 25 mg & $25 \mathrm{mg}$ & 62 & $18 \mathrm{~mm} \mathrm{Hg}^{3}$ & 34 \\
\hline Perindopril/Amlodipin & $\begin{array}{l}\text { Coveram }^{\circledR} \\
\text { Tbl. à 10/10 mg }\end{array}$ & $1 \mathrm{Tbl}$. & 333 & $45 \mathrm{~mm} \mathrm{Hg}^{4}$ & 74 \\
\hline $\begin{array}{l}\text { Valsartan/ } \\
\text { Amlodipin/ HCT }\end{array}$ & $\begin{array}{l}\text { Exforge }^{\circledR} \\
\text { Tbl. à 160/10/25 mg }\end{array}$ & $1 \mathrm{Tbl}$. & 526 & $40 \mathrm{~mm} \mathrm{Hg}^{5}$ & 131 \\
\hline
\end{tabular}

Stand Janar 2014 gemäss Arzneimittelkompendium der Schweiz.

Durchschnittliche Blutdrucksenkung über 24h nach Abzug des Plazebo Effekts (zusammengefasst in [3]).

Gemäss Fachinformation Rasilez HCT ${ }^{\circledR}$ im Arzneimittelkompendium, Rubrik Eigenschaften/Wirkungen, kein Abzug des Plazebo Effekts.

Open-label Studie von Patienten mit Grad III Hypertonie [5].

Gemäss Fachinformation Exforge HCT ${ }^{\circledR}$ im Arzneimittelkompendium, Rubrik Eigenschaften/Wirkungen, kein Abzug des Plazebo Effekts. 
der Sartane verschoben hat. Eine Therapie mit Valsartan ist heute bei gleicher blutdrucksenkender Wirkung und günstigerem Nebenwirkungsprofil nicht mehr teurer als eine Therapie mit Lisinopril. Demgegenüber gibt es aber Hinweise, dass ACE-Hemmer den ARB bezüglich Senkung der Gesamtmortalität überlegen sind [4]. Auf jeden Fall ist die Wahl zwischen ACE-Hemmer und ARB heute keine Preisfrage mehr, und die Guidelines müssten bezüglich Bevorzugung von ACE-Hemmern überdacht werden.

Grosse Preisunterschiede gibt es im Vergleich zwischen neueren Substanzen, die noch einen Patentschutz haben, und älteren Substanzen, von denen bereits Generika auf dem Markt sind. So ist der Direkte Renin-Inhibitor (DRI) Aliskiren bei gleicher Wirkung rund 8-mal teurer als Hydrochlorithiazid. Aliskiren sollte deshalb nur bei Unverträglichkeit von anderen Substanzen und nicht First-line eingesetzt werden. Auch bei den fixen Kombinationen, welche Vorteile bezüglich Compliance haben, gibt es grosse Unterschiede. Die fixe Kombination Perindopril/Amlodipin sticht dabei mit einem besonders guten Preis-Leistungs-Verhältnis heraus.

Welche Substanzklasse oder Kombination letztendlich primär zum Einsatz kommt, kann jeder Grundversorger in Anbetracht obengenannter Aspekte und aufgrund eigener Erfahrungen selber entscheiden.

\section{Interessenkonflikte}

Der Autor hat keine Interessenskonflikte im Zusammenhang mit diesem Beitrag deklariert.

\section{Literatur}

1 Schweizerische Hypertonie Gesellschaft www.swisshypertension.ch

2 Wisloff T, Slevmer RM, Halvorsen S, Fretheim A, Norheim OF, Kristiansen IS. Choice of generic antihypertensive drugs for the primary prevention of cardiovascular disease - a cost-effectiveness analysis. BMC Cardiovascular Disord. 2012;4(12):26.

3 Law MR, Wald NJ, Morris JK, Jordan RE. Value of Iwo dose combination treatment with blood pressure lowering drugs: analysis of 354 randomised trials. BMJ. 2003;326(7404):1427

4 van Vark LC, Bertrand M, Akkerhuis KM, Brugts JJ, Fox K, Mourad JJ, Boersma E. ACE-inhibitors reduce mortality in hypertension: a meta-analysis of randomized clinical trials of renin-angiotensin-aldosterone system inhibitors involving 158998 Patients. Eur Heart J. 2002;33(16):2088-971.

5 Hatala R, Pella D, Hatalová K, Šidlo R. Optimization of blood pressure treatment with fixed-combination perindopril/amlodipine in patients with arterial hypertension. Clin Drug Investig. 2012;32(9):603-12.
Korrespondenz:

Dr. med. et phil. nat. Damian N. Meli

Berner Institut für Hausarztmedizin

Medizinische Fakultät der Universität Bern

Gesellschaftsstrasse 49

3012 Bern

damian.meli[at]biham.unibe.ch 